\title{
Evidence-Based Medicine in Pediatric Endocrinology and Diabetes
}

\author{
Gary Butlera,b, Carrie Williamsª, ${ }^{a}$, Lee Hudson ${ }^{a, b}$ and Stephen O'Riordanc \\ anniversity College London Hospital, London, UK \\ bInstitute of Child Health, University College London, UK \\ 'Cork University Hospital, Cork, Ireland
}

\begin{abstract}
The principal themes in this year's chapter are hypoglycemia, its problems and prevention, strategies to improve bone health and growth, and hyperandrogenism, new insights into the diagnosis and treatment together with reports of novel uses of growth hormone. Although the studies selected are among the best we identified, the challenges of obtaining high quality clinical evidence are there to see. Important consensus documents released include the American Diabetes Association statement about transitional care [1] and continuous glucose monitoring [2]. New information about the longer term safety of growth hormone treatment is gradually emerging albeit piecemeal at the present time (see chapter on Growth and Growth Factors) [3, 4], but as yet there is not enough high quality evidence to make it into this Yearbook chapter. However, when concerns are raised, we need to be reminded of why we use such a treatment to improve short stature [5]. As ever, what is presented is a small selection from an outstandingly good publication year, selected principally to inform and guide your everyday clinical practice.
\end{abstract}

\section{Mechanism of the year \\ Keeping the $\beta$ cell going}

\section{Teplizumab for the treatment of type 1 diabetes (Protégé Study): 1-year results from a randomized placebo-controlled trial}

Sherry N, Hagopian W, Ludvigsson J, Jain SM, Wahlen J, Ferry RJ Jr, Bode B, Aronoff S, Holland S, Carlin D, King KL, Wilder RL, Pillemer S, Bonvini E, Johnson S, Stein KE, Koenig S, Herold KC, Daifotis AG

General Hospital for Children, Boston, MA, USA

nsherry@partners.org

Lancet 2011;378:487-497

Background: Small studies have suggested that short treatments with anti-CD3 monoclonal antibodies (mutated to reduce $\mathrm{Fc}_{\mathrm{C}}$ receptor binding) may preserve $\beta$-cell function and decrease insulin needs in patients with recent-onset type 1 diabetes. This phase 3 trial aims to assess the safety and efficacy of teplizumab, one such anti-CD3 monoclonal antibody.

Methods: The paper describes interim data from the first year of this 2-year trial. Patients, aged 8-35 years, who had been diagnosed with type 1 diabetes for 12 weeks or fewer were enrolled and treated at 83 clinical centers in North America, Europe, Israel, and India. Participants were allocated (2:1:1:1 ratio) by an interactive telephone system, according to computer-generated block randomization, to receive one of three regimens of teplizumab infusions (14-day full dose, 14-day low dose, or 6-day full dose) or placebo at baseline and at 26 weeks. The primary composite outcome was the percentage of patients with insulin use of $<0.5 \mathrm{U} / \mathrm{kg} /$ day and glycated hemoglobin $\mathrm{A}_{1 \mathrm{c}}\left(\mathrm{HbA}_{1 \mathrm{c}}\right)$ of $<6.5 \%$ at 1 year. Analyses included all patients who received at least one dose of study drug. As this study is still underway, patients and study staff remain masked through to study closure.

Results: 513 of 763 patients screened were randomized to receive 14-day full-dose teplizumab $(\mathrm{n}=209)$, 14-day low-dose teplizumab $(\mathrm{n}=102), 6$-day full-dose teplizumab $(\mathrm{n}=106)$, or placebo $(\mathrm{n}=99)$. Two patients in the 14-day full-dose group and 1 patient in the placebo group did not start treatment, leaving 513 patients eligible for efficacy analyses. The primary outcome did not differ between groups at 1 year: $19.8 \%$ (41/207) in the 14-day full-dose group, $13.7 \%$ (14/102) in the 14-day low-dose group, $20.8 \%(22 / 106)$ in the 6 -day full-dose group, and $20.4 \%(20 / 98)$ in the placebo group. $5 \%(19 / 415)$ of 
patients in the teplizumab groups were not taking insulin at 1 year, compared with no patients in the placebo group at 1 year $(\mathrm{p}=0.03)$. Similar proportions of patients had adverse events across the four study groups (99\% in the teplizumab groups vs. $99 \%$ in the placebo group) and serious adverse events (10 vs. $9 \%$ ). The most common clinical adverse event in the teplizumab groups was rash (53 vs. $20 \%$ in the placebo group).

Conclusion: Findings of exploratory analyses suggest that future studies of immunotherapeutic intervention with teplizumab might have increased success in prevention of a decline in $\beta$-cell function (measured by C-peptide) and provision of glycemic control at reduced doses of insulin if they target patients early after diagnosis of diabetes and especially children aged 8-11 years.

The Protégé Study is a very impressive well-designed, large, multicenter ( 83 centers), randomized, double-blind, placebo-controlled trial. Although the interim primary outcome, presented above, does not differ between treatment and placebo groups, it is definitely worth taking the time to delve a little deeper into this study as the post-hoc analysis has some interesting findings. At any given $\mathrm{HbA}_{1 \mathrm{c}}$ threshold, the authors found a greater percentage of patients in the 14-day full-dose group achieved that threshold at lower insulin doses than in the placebo group at all stages of the study. This was particularly the case for children and for adults recently diagnosed with type 1 diabetes. This study is pushing the frontiers of diabetes care both at clinical and experimental levels. It highlights the importance of including children in large, multicenter, randomized, double-blind, placebocontrolled trials and the importance of translational research linking bench to bedside. This study not only supports the need for further studies investigating this fascinating and promising mechanism, it also serves as a reminder of the importance of choosing the most appropriate primary outcome for any study.

\section{New paradigms \\ Building bones}

\section{Influence of a 3-year exercise intervention program on fracture risk, bone mass, and bone size in prepubertal children}

Lofgren B, Detter F, Dencker M, Stenevi-Lundgren S, Nilsson JA, Karlsson MK

Clinical and Molecular Osteoporosis Research Unit, Department of Orthopaedics, Lund University, Skane University Hospital, Malmo, Sweden

bjarne.lofgren@med.lu.se

J Bone Miner Res 2011;26:1740-1747

Background: Previous publications showing that exercise during childhood can improve bone mineral density have tended to follow up after short periods ( $<12$ months) and have reported other endpoints than actual fracture rates. There has been debate as to whether an increased risk of fracture from moderate exercise regimes outweighs the benefits of bone mineral density accrual.

Methods: Fractures were registered prospectively in 446 boys and 362 girls in an intervention group of an additional 40 min exercise per day over the 3-year study period, and 807 boys and 780 girls in a control group following a standardized national program of 60 min per week. A subgroup of 76 male and 48 female cases aged 7-9 years and 55 males and 44 female controls in the same age range had serial measurements of lumbar and hip bone mineral content as well as bone size using DEXA prospectively over 3 years. Presence of a fracture was also collected prospectively for all children from local hospital data. Results: There were 108 fractures during the study period. Rate ratio of fractures between cases and controls was 1.08 (95\% CI 0.71, 1.62) with no gender differences. There were no differences at baseline in age, anthropometrics, or bone traits between cases and controls. The mean annual gain in the intervention group in lumbar spine BMC was 0.9 SD higher in girls and 0.8 SD higher in boys (both $\mathrm{p}$ $<0.001$ ) and in the third lumbar vertebra width 0.4 SD higher in girls and 0.3 SD higher in boys (both $\mathrm{p}<0.05)$ than in control children.

Conclusion: A moderately intense 3-year exercise program compared with standard school ones can increase bone mineral content and possibly bone size without increasing short-term fracture risk. 
This paper and the one below share the theme of the importance of childhood and adolescence in the acquisition of longer-term bone health but look separately at an intervention to promote bone strength in childhood and a potential treatment for those with anorexia nervosa-related osteopenia.

In the study of Lofgren et al., both controls and cases received the same type of physical education, but for more regular and frequent periods than usual. The consistency of duration of physical education ensured was measured with accelerometers in both groups. Differences in extracurricular physical activity were ascertained and accounted for through questionnaires. Important baseline and follow-up analysis was made looking at changes in pubertal stage and body composition with control for the impact of both as they could potentially be confounders on evaluation of bone mineral density and bone size between groups. Furthermore, in addition to the increases in bone mineral content associated with greater physical activity, in both males and females, vertebral size was increased in those who received higher levels of regular physical education. The authors argue that this may provide additional benefits to bone strength alongside greater bone mineral content. This might be especially important in the vertebral region where crush fractures are a difficult, aged-related later-life pathology [6]. The lack of difference in fracture rates between those in a more intensive level of physical activity regimen reassures concerns over short-term negative effects of more intensive activity regimens. Interestingly, girls in with higher level of activities saw significantly bigger yearly increases in body fat. A limitation of this paper is the small number of children finally recruited into the detailed study from a larger proportion of children receiving the intervention and control across the four schools, but most likely reflects the multitude of difficulties facing all researchers when recruiting children into studies. Ultimately this paper adds to a number of health benefits associated with adequate and regular exercise for all children [7-9] and perhaps particularly highlights the importance of school-based physical education.

\section{Physiologic estrogen replacement increases bone density in adolescent girls with anorexia nervosa}

Misra M, Katzman D, Miller KK, Mendes N, Snelgrove D, Russell M, Goldstein MA, Ebrahimi S, Clauss L, Weigel T, Mickley D, Schoenfeld DA, Herzog DB, Klibanski A

Neuroendocrine Unit, Massachusetts General Hospital and Harvard Medical School, Boston, MA, USA

mmisra@partners.org

J Bone Miner Res 2011;26:2430-2438

Background: Underweight and amenorrhea are associated with reduced bone mineral density (BMD) and accrual of BMD in anorexia nervosa, as a result of nutritional and hormonal abnormalities. Previous studies of oral estrogen supplementation had not shown improvements in BMD.

Methods: 110 girls with a diagnosis of anorexia nervosa were recruited with 40 normal-weight controls. Mature girls with bone age $\geq 15$ years (96) were randomized to either transdermal $100 \mu \mathrm{g}$ of $17 \beta$-estradiol (with cyclic progesterone) or transdermal placebo for 18 months. Immature girls with bone age $\leq 15$ years were randomized to low-dose incremental oral ethinylestradiol ( $3.75 \mathrm{mg}$ daily from 0 to 6 months, $7.5 \mathrm{mg}$ from 6 to 12 months, $11.25 \mathrm{mg}$ from 12 to 18 months) or placebo for 18 months. Participants were assessed by dual-energy X-ray absorptiometry (DEXA) at 6,12 and 18 months. Participants and assessors were blind to intervention or control status (double-blind).

Results: At baseline, in both immature and mature participants, spine and hip BMD did not differ between cases provided with estrogen compared to those with placebo. Spine and hip BMI Z-scores increased over time in those treated with estrogen compared with placebo.

Conclusion: Physiological estradiol replacement increases spine and hip BMD in girls with anorexia nervosa.

The authors report a double-blind RCT which brings some hope for treatment of adolescent females with prolonged periods of underweight and persistent amenorrhea secondary to anorexia nervosa. Although weight restoration is the best treatment for improving (or least preventing deterioration in) bone mineral density consequent to underweight [10], a significant number of patients fail to achieve this, or if it happens it is very delayed [11]. Results from previous RCTs using oral estrogen have been disappointing, showing no benefit [12-14]. The authors hypothesize that the physiologi- 
cal doses of oral estrogen given in this study avoid suppression of IGF-1 secretion and the consequent reduced impact on bone formation which may occur with higher doses of estrogen therapy. The study found no effect on body mass or composition, an important finding when prescribing for patients with anorexia. The authors did not report incidence of side effects of sudden administration of estrogen to postmenarchal patients (such as mood disturbances). The study had a high attrition rate (of 150 cases and controls originally recruited, only 90 completed the trial). The long-term safety profile of estrogen in this context also remains unknown, especially in the immature group. The authors used oral progesterone to counter the possible late effects of unopposed estrogen activity in those with higher doses by transdermal route.

Finally, as with so many studies of bone mineral density in children and adolescence, this study and the one above provide detailed analysis of measured bone density which can only be used as a proxy for future fracture risk. It is important to note that neither of them are currently able to provide differences in actual long-term fracture rates between controls and cases. Thus longer-term studies are indeed essential to defend the relative costs and risks of both implementing public interventions to promote bone health and prescribing hormonal treatments for those viewed most at risk, such as those with anorexia nervosa.

\author{
New hope \\ Getting better at preventing hypos
}

\title{
Sensor-augmented pump therapy for $\mathrm{HbA}_{1 \mathrm{c}}$ reduction (STAR 3) study: results from the 6-month continuation phase
}

Bergenstal RM, Tamborlane WV, Ahmann A, Buse JB, Dailey G, Davis SN, Joyce C, Perkins BA, Welsh JB, Willi SM, Wood MA, STAR 3 Study Group

International Diabetes Center at Park Nicollet, Minneapolis, MN, USA

richard.bergenstal@parknicollet.com

Diabetes Care 2011;34:2403-2405

Background: This study examined the effects of crossing over from optimized multiple daily injection (MDI) therapy to sensor-augmented pump (SAP) therapy for 6 months, and the sustained effects of 18 months' of SAP usage.

Methods: This study was a 6-month, single-crossover continuation phase of the Sensor-Augmented Pump Therapy for $\mathrm{HbA}_{1 \mathrm{c}}$ Reduction (STAR 3) trial which provided SAP therapy to 420 patients after completing the 1-year randomized study. Change in $\mathrm{HbA}_{1 \mathrm{c}}$ was the primary outcome in the crossover group.

Results: The $\mathrm{HbA}_{1 \mathrm{c}}$ values were initially lower in the continuing-SAP group than in the crossover group (7.4 vs. $8.0 \%, \mathrm{p}<0.001)$. $\mathrm{HbA}_{1 \mathrm{c}}$ values remained lower in the SAP group. After 3 months on the SAP system, $\mathrm{HbA}_{1 \mathrm{c}}$ decreased to $7.6 \%$ in the crossover group $(\mathrm{p}<0.001)$ and there was a sustained and significant decrease in both adult and pediatric groups $(\mathrm{p}<0.05)$.

Conclusions: Converting from optimized MDI to SAP therapy allowed for rapid and safe $\mathrm{HbA}_{1 \mathrm{c}}$ reductions. Glycemic benefits of SAP therapy were sustained at 18 months in children and adults.

\section{Prevention of hypoglycemia by using low glucose suspend function in sensor-augmented pump therapy}

Danne T, Kordonouri O, Holder M, Haberland H, Golembowski S, Remus K, Blasig S, Wadien T, Zierow S, Hartmann

R, Thomas A

Children's Hospital on the Bult, Hannover, Germany

danne@hka.de

Diabetes Technol Ther 2011;13:1129-1134

Background: This study reports on the severe hypoglycemic episodes as a barrier for achieving optimal glycemic control. Sensor-augmented pump (SAP) therapy with insulin in combination with a novel mechanism of automatic insulin shutoff (low glucose suspend (LGS)) can be used to prevent and reduce 
hypoglycemia. This prospective study investigated the effect of the LGS on the frequency of hypoglycemia in children and adolescents with type 1 diabetes in routine clinical practice.

Methods: 21 patients with type 1 diabetes $(10.8 \pm 3.8$ years old, duration of diabetes $5.9 \pm 3.0$ years, pump therapy for $3.7 \pm 1.7$ years, glycated hemoglobin level $7.8 \pm 1.1 \%$ ) from three pediatric centers used the Paradigm Veo system during two subsequent time periods: SAP without LGS for 2 weeks and then SAP with LGS activated for 6 weeks. The primary aim was to assess the frequency of hypoglycemic episodes when using the LGS feature with an insulin delivery shutoff of a maximum of $2 \mathrm{~h}$ at a sensor glucose level $<70 \mathrm{mg} / \mathrm{dl}(3.9 \mathrm{mmol} / \mathrm{l})$.

Results: In total, 1,298 LGS alerts occurred ( $853<5 \mathrm{~min}) .42 \%$ of LGS activations ( $>5 \mathrm{~min}$ ) lasted $<30$ min, whereas $24 \%$ had a duration of $2 \mathrm{~h}$. The number of hypoglycemic excursions (average/day) was reduced during SAP + LGS (<70 mg/l, $1.27 \pm 0.75$ vs. $0.95 \pm 0.49, \mathrm{p}=0.010 ; \leq 40 \mathrm{mg} / \mathrm{dl}, 0.28 \pm 0.18$ vs. $0.13 \pm 0.14, \mathrm{p}=0.005$ ) as was the time spent in hypoglycemia (average minutes/day, $101 \pm 68$ vs. 58 $\pm 33, \mathrm{p}=0.002)$ without significant difference in the mean glucose level $(145 \pm 23 \mathrm{vs} .148 \pm 19 \mathrm{mg} / \mathrm{dl})$. While LGS was activated, no episodes of diabetic ketoacidosis or severe hyperglycemia were observed. Conclusions: This study provides evidence that SAP with LGS reduces the frequency of hypoglycemia without compromising safety.

Continuous glucose monitoring (CGM) has been an exciting evolving theme through the EBM chapter over the last 3 years [15]. Both of these new studies focus on the utility of continuous glucose monitoring (CGM) in children and adults with type 1 diabetes. Both studies have a good study design and sound evidence-based methodology. Bergenstal's study is a single-crossover continuation phase of 420 subjects who completed the 1-year randomized STAR 3 study $[16,17]$. The trial results of STAR 3 showed a $1 \%$ reduction in $\mathrm{HbA}_{1 \mathrm{c}}$ in adults and $0.5 \%$ reduction in children over 1 year on the SAP compared with MDI. This 6-month follow-up study reports the effects of crossing over from optimized MDI to SAP. Bergenstal et al. report safe, rapid and sustained reductions in $\mathrm{HbA}_{1 \mathrm{c}}$ in both adults and children. This study highlights the importance of translating clinical trials to clinical practice and allows us to provide good evidence to guide our day-to-day management of all adults and children with type 1 diabetes.

The second study by Danne et al. is a multicenter prospective study, assessing 21 children with type 1 diabetes using SAP for 2 weeks with the LGS feature turned off followed by 6 weeks with the LGS feature activated. The Paradigm Veo is the first insulin pump equipped with a low glucose suspend (LGS) feature which leads to an interruption in the supply of insulin for a period of up to $120 \mathrm{~min}$. This occurs when the glucose value falls below an adjustable hypoglycemia threshold (set by the patient and/or healthcare professional). If the patient does not respond to the alert and the insulin is automatically suspended. After the LGS is triggered, if the patient fails to respond by resuming insulin delivery, insulin suspension will last for $120 \mathrm{~min}$, after which insulin delivery will be automatically resumed for $4 \mathrm{~h}$. Danne et al. report that the LGS feature reduces the frequency of hypos without compromising pump safety. This is a major finding in pediatric diabetes and a very useful tool in management of nocturnal hypoglycemia and hypoglycemia unawareness, particularly in young children ( $<5$ years). Both studies highlight the importance of larger multicenter trials with realistic outcomes, which include children so this important evidence is also available for pediatric endocrinologists around the globe. 


\section{The effect of recurrent severe hypoglycemia on cognitive performance in children with type 1 diabetes: a meta-analysis}

Blasetti A, Chiuri RM, Tocco AM, Di Giulio C, Mattei PA, Ballone E, Chiarelli F, Verrotti A

Department of Pediatrics, University of Chieti, Chieti, Italy

ablasetti@tiscalinet.it

J Child Neurol 2011;26:1383-1391

Background: The aim of this study was to investigate the extent of cognitive impairment in children with type 1 diabetes with recurrent severe hypoglycemia, using meta-analysis to synthesize data across studies.

Methods: 441 children with diabetes and recurrent severe hypoglycemia and 560 children with diabetes and without recurrent severe hypoglycemia were included in this meta-analysis.

Results: Children with type 1 diabetes and recurrent severe hypoglycemia had lower performance than type 1 diabetes children without severe hypoglycemia only in some cognitive domains: learning, memory, intelligence and verbal fluency/language. Greater impairment was found in memory and learning. Motor speed was not impaired.

Conclusions: This study confirms the hypothesis that recurrent severe hypoglycemia has a selective negative effect on the children's cognitive functions.

\section{Effective treatment of hypoglycemia in children with type 1 diabetes: a randomized controlled clinical trial}

McTavish L, Wiltshire E

Department of Diabetes and Endocrinology, Capital and Coast District Health Board, Wellington, New Zealand Pediatr Diabetes 2011;12:381-387

Background: This study aimed to identify the most effective of four oral treatments for hypoglycemia in children with type 1 diabetes using a weight-based protocol during a diabetes camp.

Methods: At children's diabetes camp, the treatment of hypoglycemia was randomized to one of the four treatments, randomly assigned for each episode using a sealed envelope: glucose tablets, jellybeans, orange juice, and sugar mints. The appropriate carbohydrate dose was calculated for each child for each treatment $(0.3 \mathrm{~g}$ carbohydrate $/ \mathrm{kg})$ and provided to camp leaders. Blood glucose was measured at $0,2,5,10$, and $15 \mathrm{~min}$ and symptoms recorded.

Results: 191 hypoglycemia episodes were recorded in 39 children (1-12 episodes per child), with 2 episodes excluded because of protocol violations. 52 episodes were treated with glucose tablets, 45 with jellybeans, 44 with juice, and 48 with sugar mints. Change in glucose at $10(\mathrm{p}=0.034)$ and $15 \mathrm{~min}(\mathrm{p}$ $=0.005)$ and glucose at $15 \mathrm{~min}(\mathrm{p}=0.026)$ were significantly different between treatment groups, with jellybeans produced the lowest and slowest response. Glucose tablets did not differ significantly from juice or sugar mints. Symptoms occurred in 112 episodes, with a median time to symptom resolution of $12 \mathrm{~min}$ (interquartile range $8-15 \mathrm{~min}$ ).

Conclusions: Jellybeans are less effective treatment for hypoglycemia than the other three treatments. Glucose tablets, sugar mints and orange juice are equally effective. Treatment with $0.3 \mathrm{~g} / \mathrm{kg}$ of carbohydrate (excluding jellybeans) effectively resolved hypoglycemia in most children, with 15 min often required to normalize blood glucose.

These two studies report good evidence on a well-recognized theme: hypoglycemia in children with type 1 diabetes. The first study is a meta-analysis and the second a randomized control trial.

Blasetti et al. use a meta-analysis to generate these data, however these results must be considered with caution taking into account factors such as small sample sizes, the different definitions of severe hypoglycemia, and the variety of neuropsychological tests used. On the other hand, the overall hypothesis is confirmed that in type 1 diabetes recurrent severe hypoglycemia has a selective negative effect on the children's cognitive functions. 
McTavish et al. report four treatment options that were used randomly at children's diabetes camps: glucose tablets, jellybeans, orange juice, and sugar mints. All were effective and rapid using a $0.3-\mathrm{g} /$ $\mathrm{kg}$ equivalent carbohydrate dose protocol. Jellybeans were least effective and there was a trend towards the need for repeat treatments with jellybeans to correct hypoglycemia, which was not present with the other treatments.

Both these studies provide practical data on everyday events in the lives of children with type 1 diabetes: diabetes camps and treatment of hypoglycemia. These important but perhaps small studies are warranted to lead our clinical practice with sound evidence.

\section{Concepts revised \\ Metformin in PCOS: angel or demon?}

\section{Effects of metformin in adolescents with polycystic ovary syndrome undertaking lifestyle therapy: a pilot randomized double-blind study}

Ladson G, Dodson WC, Sweet SD, Archibong AE, Kunselman AR, Demers LM, Lee PA, Williams NI, Coney P, Legro RS

Department of Obstetrics and Gynecology, Meharry Medical College, Nashville, TN, USA

Fertil Steril 2011;95:2595-2598 e1-6

Background: A good evidence base for metformin in obese adolescents with PCOS is lacking.

Methods: 22 females (13-18 years) meeting an agreed (NIH/NICHD) PCOS definition were randomized to treatment with $500 \mathrm{mg}$ metformin four times per day (stepped up over 5 days) or placebo. Treatment regimen was double blinded. Both groups received a combined diet and exercise lifestyle intervention over 6 months. Participants were assessed monthly over a 6-month period.

Results: Baseline ages and BMI were similar in intervention and control groups. A significant decrease in serum testosterone, free androgen index was seen at 3 and 6 months in the metformin group compared with baseline. There was significant $(\mathrm{p}<0.05)$ improvement of acne at each month over the 6 months in the metformin groups compared with baseline using a standardized scoring system. Adverse gastrointestinal effects in the metformin arm were greater: abdominal pain ( $R R=3.6,95 \% \mathrm{CI} 1-12.8)$ and diarrhea $(\mathrm{RR}=4.5,95 \%$ CI $1.5-13.2)$.

Conclusions: This study could not defend the use of metformin for the management of PCOS in the light of side effects found.

The first comment to make is an important caveat: this study failed to recruit sufficient participants to meet power calculations. The researchers initially powered their RCT to detect a $25 \%$ absolute difference in serum testosterone levels based on a log-normal distribution and also for a $15 \%$ dropout rate, requiring 50 participants in each arm. Final numbers were only 11 participants in each arm. Thus the small numbers in this study significantly limited power, so much so that in the mean differences after 6 months (metformin-placebo) for over 50 measured outcomes in the trial, only the AUC glucose reached statistical significance. Despite this, the authors used a rigorous definition of PCOS a robust double-blind placebo-controlled RCT design. The results presented graphically in the paper show greater decreases in serum testosterone, free androgen index compared with placebo. They discuss that lack of interest and the burden of the study were the key reasons for the failed recruitment, an important insight into the difficulties faced in research in young people with obesity [18]. The paper reminds us of the importance of side effects of metformin use, which lead the authors to conclude that use of metformin may not be justifiable - although they did not report on adherence during the trial. The authors did not indicate whether or not the metformin formulation used in the trial was slow-release which may balance side effects and increase compliance. The reduction in acne (measured using standardized pro forma for assessment of number of open and closed comedones) at each month might be an important benefit for young people that potentially could improve compliance in the clinical setting. Both cases and controls also received a lifestyle intervention, a context that may be difficult to provide in clinical practice. 


\section{Final adult height in children with congenital adrenal hyperplasia treated with growth hormone}

Lin-Su K, Harbison MD, Lekarev O, Vogiatzi MG, New MI

Department of Pediatric Endocrinology, Weill Medical College of Cornell University, New York, NY, USA

J Clin Endocrinol Metab 2011:96:1710-1717

Background: This study aimed to examine whether $\mathrm{GH}$ alone or in combination with an $\mathrm{GnRH}$ analogue (LHRH analogue) improved adult height in patients with congenital adrenal hyperplasia (CAH) due to 21-hydroxylase deficiency as adult height is usually well below midparental/target height.

Methods: This nonrandomized, prospective study included 34 patients (19 males, 15 females) with CAH, treated with $\mathrm{GH}$ as they were predicted to be $>2$ SD below their midparental target height or $>2 \mathrm{SD}$ below the population mean. GnRH analogue was also given to 27 patients (16 males, 11 females) for precocious puberty, mean duration was $3.7 \pm 1.7$ years. The mean duration of GH treatment was $5.6 \pm$ 1.8 years in males and $4.5 \pm 1.6$ years in females.

Results: Males achieved a significantly taller adult height $(172.0 \pm 4.8 \mathrm{~cm})$ than predicted $(162.8 \pm 7.7$ $\mathrm{cm})(\mathrm{p}<0.00001)$. Females also became significantly taller $(162.2 \pm 5.3 \mathrm{~cm})$ than predicted $(151.7 \pm$ $5.2 \mathrm{~cm})(\mathrm{p}<0.00001)$. The mean height gain was $9.2 \pm 6.7 \mathrm{~cm}$ in males and $10.5 \pm 3.7 \mathrm{~cm}$ in females. Conclusions: This study demonstrates the possible height gains that can be achieved with GH alone or in combination with GnRH analogue in patients with CAH.

The degree of reduction in adult height in patients with $\mathrm{CAH}$ and its causes are well described in this paper, including the tendency to develop central precocious puberty as a result of the chronic hyperandrogenism. This paper presents an important comprehensive extension of the only study that addresses the long-term use of GH in the $\mathrm{CAH}$ population, and hence the inclusion in this chapter, despite the study not using a randomized control design. Inclusion criteria were typical to those encountered in routine practice: bone age of at least 6 years and >1 SD ahead of chronological age; adult height prediction by the Bayley-Pinneau method of at least 2 SD below midparental target height or at least 2 SD below the population mean (males $177 \pm 7 \mathrm{~cm}$, females $163 \pm 6 \mathrm{~cm}$ ), and with open epiphyses (bone age under 13 years in girls and 15 years in boys), and hence on that count, a very useful exposition of an attempt to rescue growth in these children we often see with difficult $\mathrm{CAH}$. The greater height gain in females over males was explained by the worse initial height prognosis in this group of males. It is important to note that GnRH analogue did not appear to add any clear height gain benefit to GH therapy, so the authors stress that the decision to treat with GnRH analogue should be based on the age of the patient and the social impact of precocious puberty rather than for the sole purpose of increasing the gain in height. This very positive report now gives grounds for further exploration of this treatment approach.

This paper and the RCT of GH in short children with X-linked hypophosphatemic rickets by Zivicnjak et al. (see chapter by Cianfarani) each present evidence of GH efficacy with differing evidence bases, neither perfect. Both are relatively small, one long-term to adult height, and one a medium-term $\mathrm{RCT}$, and both are for clinical indications not currently bearing a license for GH treatment. However, they both provide the necessary groundwork to take trials forward to the next stage. 


\section{Prospective study on the prevalence and associated risk factors of cryptorchidism in 6,246 newborn boys from Nice area, France}

Wagner-Mahler K, Kurzenne JY, Delattre I, Berard E, Mas JC, Bornebush L, Tommasi C, Boda-Buccino M, Ducot B, Boulle C, Ferrari P, Azuar P, Bongain A, Fenichel P, Brucker- Davis F

Pediatrics Department, CHU, Nice, France

Wagner.k@pediatrie-chulenval-nice.fr; wagner@chu-nice.fr

Int J Androl 2011;34:e499-510

Background: Cryptorchidism is the most common congenital malformation in boys. The incidence appears to vary markedly between studies, and it remains unclear whether the incidence is rising, largely due to methodological limitations in previous studies. Therefore this study aimed to assess the incidence and risk factors of cryptorchidism in the Nice area.

Methods: A prospective study was conducted at two maternity wards over a 3-year period. All boys born $\geq 34$ weeks after the last menstrual period during the study period were screened. The methodology was strict with examination at birth, 3 and 12 months by the same pediatrician. Two carefully matched controls were included for each case. Information on child and parents (including lifestyle, medical history and pregnancy) was recorded using medical chart information and self-administered questionnaires.

Results: Of the 6,246 boys born within the duration of the study, a total of 102 were born with cryptorchidism (prevalence 1.6\%, 95 included in the study). Half were still cryptorchid at 3 and 12 months with $10 \%$ recurrence of cryptorchidism at 12 months, justifying long-term follow-up. Instrumental delivery, inguinal hernia and urogenital malformations, particularly micropenis and paternal history of cryptorchidism, were associated with cryptorchidism at birth. Results suggested that maternal exposure to antirust or phthalates could be a risk factor, whereas eating fruits daily seemed somewhat protective.

Conclusions: The prevalence of cryptorchidism in this study is on the lower side compared with estimates from other countries. Cryptorchidism was found to be associated with both familial and environmental risk factors.

Cryptorchidism is the most common congenital malformation in boys, with estimated incidence varying between 0.9 and $9 \%$. Various authors have suggested a variety of environmental exposures as risk factors for cryptorchidism, and previous studies have attempted to quantify the influence of these exposures. This population-based case-control study is well designed and conducted to overcome limitations of previous studies including those relating to definition and diagnosis. The assessment of children was highly standardized, both between centres and between examinations at different ages. Follow-up rates were very good, between 90 and $96 \%$ throughout the study.

At 12 months of age, $41 \%$ had spontaneously resolved, the overwhelming majority of these before 3 months ( 1 case resolved after 3 months but before 12 months). In addition, this paper demonstrates well the need for follow-up of children who have had apparent resolution at 3 months as they reported a $10 \%$ recurrence rate between 3 and 12 months of age.

This paper also reports a number of apparent risk factors for cryptorchidism some already well described including a paternal history of cryptorchidism, prematurity, and some less well described including maternal exposure to antirust products and phthalates, living in a rural setting and instrumental delivery. When interpreting these findings, as indeed for all research, authors and readers alike must be cautious. Whilst some association may be true risk factors, other apparent risk factors may be serving as 'proxies' for other factors. For example, this paper found that eating fruit daily appeared to act as a protective factor for the cryptorchidism. The authors point out that this is unlikely to be a true effect, but rather may be due to 'confounding' by some other socioeconomic status-related exposure. 


\section{Treatment of androgen excess in adolescent girls: ethinylestradiol- cyproterone acetate versus low-dose pioglitazone-flutamide-metformin}

Ibanez L, Diaz M, Sebastiani G, Sanchez-Infantes D, Salvador C, Lopez-Bermejo A, de Zegher F

Endocrinology Unit, Hospital Sant Joan de Déu, University of Barcelona, Esplugues, Barcelona, Spain

libanez@hsjdbcn.org

J Clin Endocrinol Metab 2011;96:3361-3366

Background: Traditional management of the androgen excess in PCOS in postmenarchal females has been by ovarian-focused treatment using combined estrogen and progesterone medication. This study aimed to compare use of such a therapy with a regimen directed towards systemic abnormalities in metabolism and body fat storage.

Methods: An open-labeled, randomized study in postmenarchal girls of ethinylestradiol-cyproterone acetate (EE-CA) or low-dose combination of pioglitazone, flutamide, and metformin (PioFluMet) with comparison of clinical and endocrine-metabolic outcomes at 6 months.

Results: EE-CA and PioFluMet were equally as effective in reducing laboratory and clinical measures of androgen excess. Effects on fasting insulinemia; circulating cholesterol, triglycerides, C-reactive protein, high-molecular-weight adiponectin, leptin, and follistatin; carotid intima-media thickness; lean mass, and on abdominal, visceral, and hepatic fat, were significantly healthier with low-dose PioFluMet compared to EE-CA.

Conclusions: Low-dose PioFluMet compared favorably with EE-CA in adolescents with androgen excess and with no pregnancy risk. The efficacy and safety of low-dose PioFluMet remain to be studied over a longer term and in larger cohorts.

This study challenges the traditional thinking of PCOS as a disorder primarily of the ovary and the associated treatment model of ovarian suppression as route to decreasing androgen excess [19]. Ibáñez et al. propose an alternative way of thinking about PCOS which links hyperandrogenism with abnormalities of body fat storage, in particular visceral adiposity. This they argue would bring together the multiple metabolic and endocrine abnormalities associated with the condition, for example insulin resistance, as well as the chronological pathway from visceral adiposity to PCOS in postmenarche. They tested this proposal in an open-labelled randomized trial comparing traditional estrogen-progesterone therapy (EE-CA) with a combination of two metabolic antidiabetes medications (pioglitazone and metformin) with the antiandrogen flutamide (PioFluMet). Although both regimens produced comparable antiandrogen effects, the more systemic effects on metabolic risk such as fasting insulin, lipids and fat distribution were much more evident with PioFluMet. This trial was not blinded, but this seems appropriate when considering the potential dangers associated with pregnancy whilst on the PioFluMet regimen, a consideration which may also have implications for its clinical application in a postmenarchal group of women. The study does not seem to have been formally powered and numbers were small, though importantly no patients were lost to follow-up. Further study of the PioFluMet is warranted to identify longer term clinical effects in this age group. 


\section{Effect of intensive insulin therapy on the somatotropic axis of critically ill children}

Gielen M, Mesotten D, Brugts M, Coopmans W, Van Herck E, Vanhorebeek I, Baxter R, Lamberts S, Janssen JAMJL, Van den Berghe $\mathrm{G}$

Department of Intensive Care Medicine, Katholieke Universiteit Leuven, Belgium

Dieter.Mesotten@med.kuleuven.be

J Clin Endocrinol Metab 2011;96:2558-2566

Background: Intensive insulin therapy (IIT) is known to improve outcomes in the adult and pediatric intensive care unit (PICU) compared with conventional insulin therapy (CIT). IIT does not increase the anabolic hormone IGF-I in critically ill adults, but feeding in critically ill children and pediatric hormonal responses may differ. This study hypothesized that IIT reactivates the somatotropic axis and anabolism in PICU patients.

Methods: The authors performed a preplanned subanalysis of a randomized controlled trial of IIT. 369 patients who stayed in PICU for at least 3 days were included in the main analysis (study 1). 126 of these 369 patients were also included in a nested case-control study (study 2). Circulating insulin, C-peptide, GH, IGF-I, bioavailable IGF-I, IGF-binding protein (IGFBP)-1, IGFBP-3, and acid-labile subunit were analyzed upon admission and day 3. In the nested case-control study, the somatotropic axis, cortisol, and glucagon were analyzed before and after hypoglycemia.

Results: On day 3, C-peptide was 10-fold lower $(\mathrm{p}<0.0001)$ in the IIT group than in the CIT group. IIT lowered bioavailable IGF-I $(\mathrm{p}=0.002)$ and increased circulating $\mathrm{GH}(\mathrm{p}=0.04)$ and. IIT also decreased IGFBP-3 ( $\mathrm{p}=0.0005)$ and acid-labile subunit $(\mathrm{p}=0.007)$, while increasing IGFBP-1 $(\mathrm{p}=0.04)$ and the urea/creatinine ratio, a marker of catabolism $(p=0.03)$. In the nested case-control study, IGFBP-1 was increased after hypoglycemia. No change was noted in either the somatotropic axis or the counterregulatory hormones cortisol and glucagon.

Conclusion: Despite improved PICU outcome, IIT was not shown to counteract the catabolic state of critical illness. Lower bioavailable IGF-I may have been the result of suppression of portal insulin.

This group's previous study showed that IIT improved both mortality and morbidity in both infants and children admitted to PICU regardless of the cause or severity of illness [20]. In this preplanned analysis of the same RCT, they attempted to investigate the underlying mechanism. Contrary to the a priori hypothesis, this analysis found no evidence that the benefits of IIT are related to counteraction of the catabolic state by reactivation of the GH/IGF-1 axis. In contrast to expected results, this group actually found lowered levels of bioavailable IGF-1. The authors consider a number of possible mechanisms to explain their findings including (1) aggravated GH resistance, (2) induction of a counterregulatory response by hypoglycemia, and (3) suppression of portal insulin. This last possibility is supported by the low C-peptide levels found in the IIT group. So, if it is not through the reversal of catabolism, what is the mechanism by which IIT results in improved outcomes for PICU patients? The authors postulate that IIT is likely to improve outcomes by improving insulin sensitivity. This conclusion is supported by other studies, including a similar study in children with burns reviewed in last year's edition of this chapter $[15,21]$. 


\section{Responsiveness to metformin in girls with androgen excess: collective influence of genetic polymorphisms}

Diaz M, Lopez-Bermejo A, Sanchez-Infantes D, Bassols J, de Zegher F, Ibanez L

Endocrinology Unit, Hospital Sant Joan de Déu, University of Barcelona, Barcelona, Spain

Fertil Steril 2011;96:208-213 e2

Background: Metformin exerts metabolic effects at different tissue types and its effect can potentially be modified by a number of regulating genes. This study investigated the relationship between the efficacy of metformin use in girls with known androgen excess and combinations of genetic variants.

Methods: Adolescent girls with known hyperandrogenism and who were treated with metformin were recruited from a university hospital. A polymorphism score was calculated in each participant based on single nucleotide polymorphisms in the OCT1, STK11 and FTO genes, and number of repeats in the SHBG and AR genes. Participants were followed after 1 year of metformin use.

Results: 104 adolescent girls were enrolled. Mean BMI at baseline was $21.8 \pm 0.2$. All girls were postmenarchal. Both OCT1 and FTO showed no major associations at baseline or follow-up. Polymorphism scores had no association at baseline, however an increasing polymorphism score was associated with more favorable endocrine-metabolic outcomes of metformin use at the 1-year follow-up.

Conclusion: Collectively, genetic polymorphisms appear to have a positive impact on the outcomes of metformin use in postmenarchal girls with androgen excess.

As part of the evidence base for metformin in PCOS in children and adolescence, Diaz et al. examine the important question as to whether varying outcomes within young women receiving metformin is mediated by genetic differences. Metformin acts at a number of sites, and therefore a number of genes may modify its effects at the cellular level [22]. OCT1 encodes the organic cation transporter 1, a regulator of hepatic gluconeogenesis, which metformin is known to decrease [23]. Metformin also influences catalysis of AMP-activated protein kinase by serine-threonine kinase 11 (STK11) [24]. The fat mass and obesity-associated gene (FTO) is associated with degree of fat mass and insulin resistance in PCOS [25]. Variability in the number of repeats in both the sex hormone-binding globulin (SHBG) gene and in the androgen receptor (AR) gene have been hypothesized to alter the phenotype of PCOS and also the response to metformin $[26,27]$.

Diaz et al. demonstrated that these variants, either in isolation or in combination, showed little associations with baseline endocrine and metabolic features. However, more favorable metabolic responses to metformin (e.g. lower fasting insulin and body fat mass) were seen in participants with certain combinations of variants. A highly specific definition was used to identify this patient group, girls with a sequence of low birth weight, precious puberty and androgen excess. None was obese and all had the same ethnic background. Thus the findings may not be generalizable to other girls with PCOS, in other locations. However it may explain the variable effects of metformin in PCOS in the clinical setting as well as in research studies. Genetic categorization is however unlikely to be readily accessible in the clinical setting in the near future to assist clinical decision-making. 


\section{Efficacy and harms of nasal calcitonin in improving bone density in young patients with inflammatory bowel disease: a randomized, placebo- controlled, double-blind trial}

Pappa HM, Saslowsky TM, Filip-Dhima R, DiFabio D, Lahsinoui HH, Akkad A, Grand RJ, Gordon CM

Center for Inflammatory Bowel Disease, Children's Hospital Boston, MA, USA

Helen.pappa@childrens.harvard.edu

Am J Gastroenterol 2011;106:1527-1543

Background: Very few published studies have investigated therapies to improve bone health in children with inflammatory bowel disease (IBD). This study aimed to establish the safety and efficacy of intranasal calcitonin in improving bone mineral density (BMD) in young patients with IBD. In addition, the study aimed to identify additional factors that impact bone mineral accrual.

Methods: In a randomized, placebo-controlled, double-blind trial, 63 patients, aged 8-21 years, with IBD and a spinal BMD Z-score $\leq-1.0 \mathrm{SD}$ (measured by dual energy X-ray absorptiometry) were randomized to $200 \mathrm{IU}$ intranasal calcitonin $(\mathrm{n}=31)$ or placebo $(\mathrm{n}=32)$ daily. All received age-appropriate calcium and vitamin D supplementation. At 9 and 18 months, BMD was measured.

Results: Intranasal calcitonin was well tolerated. Adverse event frequency was similar in both groups and largely reversible, minor, and limited to the upper respiratory tract. The change in BMD Z-score between 0 and 9 months and 0 and 18 months did not differ between the two trial groups. In the subgroup with Crohn's disease, the spinal BMD Z-score improved between screening and 9 months in the calcitonin group $0.21(0.37)$ over the placebo group $0.15(0.5), p=0.02$. Factors associated with lower bone mineral accrual rate were: IBD severity (indicated by elevated inflammatory markers, need for surgery, hospitalization, and the use of immunomodulators) and higher daily caffeine intake. Factors favoring higher bone mineral accrual rate were: lower baseline BMD and higher baseline body mass index Z-score, improvement in height Z-score, higher serum albumin, hematocrit and iron concentration, and more hours of weekly weight-bearing activity.

Conclusions: Intranasal calcitonin is well tolerated. However, it does not offer a long-term advantage in adolescents with IBD and decreased BMD. Bone mineral accrual rate remains compromised in adolescents with IBD. Improvement in nutritional status, catch-up linear growth, control of inflammation, increase in weight-bearing activity, and lower daily caffeine intake may be helpful in restoring bone density in children with IBD.

This well-designed double-blind, placebo-controlled RCT is the first to investigate the effect of intranasal calcitonin on BMD in children with IBD. Unfortunately, it did not find any evidence to support this treatment. Only one positive finding within the subgroup of children with Crohn's disease was seen out of $32 \mathrm{p}$ value calculations. As the authors do not include a Bonferroni correction for multiple comparisons, carrying out this number of significance tests would mean that it is entirely plausible to find one significant result by chance alone. This could also be the logical explanation, given the lack of a biologically plausible reason for an effect solely in this subgroup. Despite the negative results, the robust design of this study gives directions for future studies. In order to adequately control for confounding factors, the authors have very accurately measured other factors which they found to be affecting BMD, some of which have not been adequately quantified before including the effect of weight-bearing activity, the effect of good disease control (measured via inflammation markers), and the effect of caffeine. The evidence suggests that it is these factors which may hold the key to improved BMD in such patients and indeed, perhaps where future studies should concentrate. 


\section{Testosterone and the child (0-12 years) with Klinefelter syndrome $(47, \mathrm{XXY})$ : a review}

Fennoy I

Department of Pediatrics, Columbia University Medical Center, New York, NY, USA

if1@columbia.edu

Acta Paediatr 2011;100:846-850

Background: This review aimed to explore the evidence base for considering testosterone therapy in infant and prepubertal boys with Klinefelter syndrome (KS).

Methods: The author searched major databases for articles that addressed the role of testosterone in the development of the male fetus with and without KS characterizing testicular function in KS during prepuberty.

Results: There may be an increased frequency of clinical features consistent with deficient testosterone production in infants with KS. However, results are conflicting as to whether testosterone levels are low or normal. There are no complete studies addressing the outcome of therapy in prepubertal boys.

Conclusions: Currently, the only documented benefit for testosterone therapy in these children is for the management of microphallus. There is an absence of data that directly address the risks and benefits of testosterone therapy in prepubertal boys with KS for any other parameter.

It is a great paradox that we know so little about the commonest human chromosome variation, 47, XXY. This review was one of several published in this supplement of Acta Paediatrica addressing what is currently known about Klinefelter syndrome, and what the evidence base for clinical practice is or should be [28]. The evidence for androgen deficiency in late puberty and adulthood is better known, but what about in the pre- and peripubertal boy, and indeed what about during infancy? Are variations in gonadal function during mini-puberty responsible for microphallus, or indeed cryptorchidism? The evidence placed before us is mixed, and very dependent upon the source of the subjects. Those where ascertainment is less biased (newborn population screening or antenatal diagnosis) may provide a more realistic prediction of gonadal function. So, infantile testosterone levels are probably within normal or low normal limits, as are those during childhood. In contrast, testosterone concentrations climb to upper normal in the peripubertal age range and can even be high in early puberty. This review also considers the reasons for benefits and potential disadvantages of giving exogenous testosterone to the prepubertal boy and on his testes. Theoretically, due to a possible increased sensitivity of the testis to intratesticular testosterone during this time period, testosterone treatment could even enhance the damage and limit even further the already reduced fertility prospects. So there is much work to do. This series of reviews, although primarily clinical reviews and not truly systematic reviews, significantly enhances our understanding of this condition and should be read by any pediatrician or endocrinologist working in this field.

\section{Food for thought \\ Milk and the camel without a hump?}

\section{Milk protein intake, the metabolic-endocrine response, and growth in infancy: data from a randomized clinical trial}

Socha P, Grote V, Gruszfeld D, Janas R, Demmelmair H, Closa-Monasterolo R, Subias JE, Scaglioni S, Verduci E,

Dain E, Langhendries JP, Perrin E, Koletzko B

Children's Memorial Health Institute, Warsaw, Poland

p.socha@czd.pl

Am J Clin Nutr 2011;94:1776S-1784S

Background: This study examined the influence of protein intake in infancy on serum amino acids, insulin, and the insulin-like growth factor I (IGF-I) axis and its possible relation to growth in the first 2 years of 
life as it has been suggested protein intake in early infancy is an important risk factor for later obesity. Information on potential mechanisms is very limited.

Methods: In a multicenter European double-blind randomized trial, 1,138 healthy, formula-fed infants were randomized to receive cows' milk-based infant and follow-on formulas with lower protein (1.77 and $2.2 \mathrm{~g}$ protein $/ 100 \mathrm{kcal})$ or higher protein $(2.9$ and $4.4 \mathrm{~g}$ protein/100 kcal) contents over the first year. Biochemical variables were measured at age 6 months in 339 infants receiving the lower protein formula and 333 infants receiving the higher protein formula and in a parallel group of 237 breast-fed infants.

Results: Essential amino acids, especially branched-chain amino acids, IGF-I, and urinary C-peptide:creatinine ratio, were significantly $(\mathrm{p}<0.001)$ higher in the higher protein group than in the lower protein group, and more so compared with the breast-fed group, whereas IGF-binding protein (IGF-BP) 2 was lower and IGF-BP3 did not differ significantly. Total IGF-I was significantly associated with growth until 6 months of age but not thereafter. The median IGF-I total serum concentration was $48.4 \mathrm{ng} / \mathrm{ml}$ (25th, 75th percentile: $27.2 ; 81.8 \mathrm{ng} / \mathrm{ml}$ ) in the higher protein group and $34.7 \mathrm{ng} / \mathrm{ml}(17.7$; $57.5 \mathrm{ng} / \mathrm{ml}$ ) in the lower protein group.

Conclusions: Higher protein intake stimulates the IGF-I axis and insulin release in infancy. The higher IGF-I may be associated with enhanced growth during the first 6 months of life.

This large European double-blind randomized trial was conceived to further explore mechanisms behind the development of infant obesity, higher protein intake already known to be associated with faster infant growth. The design randomized bottle-fed infants to the variable protein level intake, the comparator being a separate group of breast-fed infants. The size of this trial, despite the high dropout rate, provided sufficient power and the international element avoided the influence of local determinants on the outcome. The endocrine and metabolic parameters studied allowed for a more detailed insight to be gained into the mechanism of faster growth. Key findings include the protein induced drive of IGF-1 production, apparently separate from the GH axis (IGFBP-3 being unchanged between the groups). Insulin secretion increased too with the higher protein intake as deduced from elevated urinary C-peptide:creatinine ratios. Conversely at this age, glucose levels fell in the higher protein group, with insulin sensitivity being retained, again possibly a consequence of unchanged IGFBP-3 concentrations. The observed greater weight-for-length and BMI gains are likely to have been determined by the protein content of the infant feeds, but as the authors discuss, the opportunities for an even more detailed study, especially on amino acid metabolism were not possible, so many questions on the exact mechanism of the faster growth remain unanswered.

\section{Effect of camel milk on glycemic control and insulin requirement in patients with type 1 diabetes: 2-years randomized controlled trial}

Agrawal RP, Jain S, Shah S, Chopra A, Agarwal V

Department of Medicine, Diabetes Care \& Research Centre, SP Medical College, Bikaner, Rajasthan, India drrpagrawal@yahoo.co.in

Eur J Clin Nutr 2011;65:1048-1052

Background: Camel milk supplementation has been shown to exert a hypoglycemic effect both in an experimental rat model and previous studies from this group had shown a significant reduction in doses of insulin in type 1 diabetic patients. This was a 2-year randomized open clinical, parallel-design study and was undertaken to assess the efficacy, safety and acceptability of camel milk jointly with insulin therapy in adolescents and young adults with type 1 diabetes.

Methods: 24 patients were selected at random then randomized into two groups. Group I $(\mathrm{n}=12$; ages $14.5 \pm 8.4$ years) received usual care comprising of diet, exercise and insulin and group II $(\mathrm{n}=12$; ages $16.7 \pm 8.9$ years) additionally received $500 \mathrm{ml}$ camel milk daily. Insulin dosage was titrated weekly following blood glucose estimation.

Results: In the intervention group, the mean blood glucose decreased from $6.6 \pm 1.1$ to $5.2 \pm 0.9 \mathrm{mmol} / \mathrm{l}$, as did $\mathrm{HbA}_{1 \mathrm{c}}$ levels $(7.8 \pm 1.4$ to $5.5 \pm 0.8 \%)$. There was a fall in and insulin requirement from $32.5 \pm$ 10.0 to $17.5 \pm 12.1 \mathrm{U} /$ day $(\mathrm{p}<0.05)$, with 3 subjects reducing their insulin requirement to zero. Group I showed the expected rises in $\mathrm{HbA}_{1 \mathrm{c}}$. No adverse hypoglycemic events were noted.

Conclusions: This study suggests that camel's milk is safe and efficacious in improving glycemic control in patients with type 1 diabetes, with a significant reduction in the dosage of insulin. 
It had been previously observed by the same group that populations drinking camel's milk as part of their regular diet exhibited a low incidence of diabetes, and pilot intervention studies demonstrated improvements in diabetes control. This longer term RCT, albeit small, has indeed demonstrated significant effects on glycemic control parameters and reductions in insulin requirement actually to zero in 3/12 subjects. Camel's milk contains proteins which, being similar to insulin, may inhibit hepatic gluconeogenesis. Additionally it contains high concentrations of insulin, actually similar to human milk, but camel's milk does not undergo coagulation in the stomach thus making the insulin available for intestinal absorption. Whether there is mileage in exploring this effect further is unclear, but the principal lesson from this study is an exploration of the adaptation to the local environment and the design of a simple yet effective study design to investigate regional observations in greater depth. The good camel has now lost its hump! [29].

\section{Follow-up on Yearbooks 2010 and 2011}

\section{The effect of oxandrolone on voice frequency in growth hormone-treated girls with Turner syndrome}

Menke LA, Sas TC, van Koningsbrugge SH, de Ridder MA, Zandwijken GR, Boersma B, Dejonckere PH, de Muinck Keizer-Schrama SM, Otten BJ, Wit JM

Dutch Growth Research Foundation, Rotterdam, The Netherlands

l.a.menke@lumc.nl

J Voice 2011;25:602-610

Background: Previous studies published by this group have shown oxandrolone increases height gain, however it may also cause voice deepening in growth hormone (GH)-treated girls with Turner syndrome. This study also assessed the effect of oxandrolone on objective and subjective speaking voice frequency in GH-treated girls with Turner syndrome.

Methods: This multicenter, randomized, placebo-controlled, double-blind study enrolled 133 participants. Patients were randomized to treatment with $\mathrm{GH}\left(1.33 \mathrm{mg} / \mathrm{m}^{2} /\right.$ day $)$ combined with either placebo, low-dose oxandrolone $(0.03 \mathrm{mg} / \mathrm{kg} /$ day $)$ or conventional-dose oxandrolone $(0.06 \mathrm{mg} / \mathrm{kg} / \mathrm{day})$. Patients were treated from age 8 and estrogens were added from 12 years. Voices were recorded and subjective voice quality questionnaires were completed yearly from starting treatment until 6 months after discontinuing.

Results: Voice frequency standard deviation scores (SDS) were high for age at the baseline assessment $(1.0 \pm 1.2, \mathrm{p}<0.001)$ but normal for height. Compared with the placebo group, voices tended to become lower in the low-dose oxandrolone group $(\mathrm{p}=0.09)$ and significantly lowered in the conventional-dose oxandrolone group $(\mathrm{p}=0.007)$. At the final measurement, voice frequency SDS was still relatively high in the placebo group $(0.6 \pm 0.7, \mathrm{p}=0.002)$ but similar to healthy girls in both oxandrolone groups. Voice frequency became lower than -2 SDS in 1 patient $(3 \%)$ on low-dose oxandrolone and 3 patients $(11 \%)$ on conventional-dose oxandrolone. The percentage of patients reporting subjective voice deepening was similar between the dosage groups.

Conclusion: Untreated girls with Turner syndrome have relatively high-pitched voices. The addition of oxandrolone to GH decreases voice frequency in a dose-dependent way. Although most voice frequencies remain within the normal range, they may occasionally become lower than -2 SDS, especially on conventional doses of oxandrolone $(0.06 \mathrm{mg} / \mathrm{kg} / \mathrm{day})$.

Safety and growth outcomes from this RCT have been reviewed in this chapter in 2010 and 2011 respectively [15, 30]. Last year we described this study as 'elegant, well designed and well conducted' and indeed this year's paper provides further evidence of the elegance of the design. It provides further information on the efficacy-to-safety ratio of oxandrolone by reporting the subjective and objective frequency of the speaking voice of participants. At baseline, voice frequency was found to be higher in participants, in comparison to chromosomally normal girls of the same age. This is particularly so for girls with monosomy or isochromosome karyotypes and girls who do not experience spontaneous puberty. The study confirmed that low-dose oxandrolone has a favorable efficacy/side 
effect profile, not only increasing final height, but resulting in a slight lowering of voice frequency to levels comparable to the general population. It also verified previous findings that conventional-dose oxandrolone can result in unfavorable side effect of significantly lowering voice (>-2 SDS in $11 \%$ of participants) as well as potentially causing virilization.

As clinicians, this is study is particularly useful as the results of all three resulting publications from this RCT help us to assist our patients and their families to make fully informed decisions about treatment. It provides evidence not only on outcomes we consider important, but also those valued by our patients.

References

1. Phillip M, Danne T, Shalitin S, Buckingham B, Laffel L, Tamborlane W, Battelino T, for the Consensus Forum Participants: Use of continuous glucose monitoring in children and adolescents. Pediatr Diabetes 2012;13:215-228.

2. Peters A, Laffel L: Diabetes care for emerging adults: recommendations for transition from pediatric to adult diabetes care systems: a position statement of the American Diabetes Association, with representation by the American College of Osteopathic Family Physicians, the American Academy of Pediatrics, the American Association of Clinical Endocrinologists, the American Osteopathic Association, the Centers for Disease Control and Prevention, Children with Diabetes, The Endocrine Society, the International Society for Pediatric and Adolescent Diabetes, Juvenile Diabetes Research Foundation International, the National Diabetes Education Program, and the Pediatric Endocrine Society (formerly Lawson Wilkins Pediatric Endocrine Society). Diabetes Care 2011;34:2477-2485.

3. Savendahl L, Maes M, Albertsson-Wikland K, Borgstrom B, Carel JC, et al: Long-term mortality and causes of death in isolated GHD, ISS, and SGA patients treated with recombinant growth hormone during childhood in Belgium, the Netherlands, and Sweden: preliminary report of three countries participating in the EU SAGhE Study. J Clin Endocrinol Metab 2012;97:E213-E217.

4. Carel JC, Ecosse E, Landier F, et al: Long-term mortality after recombinant growth hormone treatment for isolated growth hormone deficiency or childhood short stature: preliminary report of the French SAGhE Study. J Clin Endocrinol Metab 2012;97:416-425.

5. Gardner M, Sandberg DE: Growth hormone treatment for short stature: a review of psychosocial assumptions and empirical evidence. Pediatr Endocrinol Rev 2011;9:579-588.

6. Gold D: The clinical impact of vertebral fractures: quality of life in women with osteoporosis. Bone 1996;18:S185-S189.

7. Janz KF, Kwon S, Letuchy EM, Eichenberger Gilmore JM, Burns TL, Torner JC, et al: Sustained effect of early physical activity on body fat mass in older children. Am J Prev Med 2009;37:35-40.

8. Brage S, Wedderkopp N, Ekelund U, Franks PW, Wareham NJ, Andersen LB, et al: Features of the metabolic syndrome are associated with objectively measured physical activity and fitness in Danish children. Diabetes Care 2004;27:21412148.

9. Ekelund U, Anderssen S, Froberg K, Sardinha L, Andersen L, Brage S: Independent associations of physical activity and cardiorespiratory fitness with metabolic risk factors in children: the European Youth Heart Study. Diabetologia 2007;50:1832-1840.

10. Hudson LD, Court AJ: What paediatricians should know about eating disorders in children and young people. J Paediatr Child Health 2012 (E-pub ahead of print).

11. Jayasinghe Y, Grover SR, Zacharin M: Current concepts in bone and reproductive health in adolescents with anorexia nervosa. BJOG 2008;115:304-315.

12. Gordon CM, Grace E, Emans SJ, Feldman HA, Goodman E, Becker KA, et al: Effects of oral dehydroepiandrosterone on bone density in young women with anorexia nervosa: a randomized trial. J Clin Endocrinol Metab 2002;87:49354941.

13. Klibanski A, Biller B, Schoenfeld D, Herzog D, Saxe V: The effects of estrogen administration on trabecular bone loss in young women with anorexia nervosa. J Clin Endocrinol Metab 1995;80:898-904.

14. Grinspoon S, Thomas L, Miller K, Herzog D, Klibanski A: Effects of recombinant human IGF-I and oral contraceptive administration on bone density in anorexia nervosa. J Clin Endocrinol Metab 2002;87:2883-2891.

15. Butler G, Williams C, O'Riordan S: Evidence-based medicine in pediatric endocrinology; in Carel JC, Hochberg Z (eds): Yearbook of Pediatric Endocrinology 2011. Basel, Karger, 2011, pp 193-214.

16. Bergenstal RM, Tamborlane WV, Ahmann A, et al, for the STAR 3 Study Group: Effectiveness of sensor-augmented insulin-pump therapy in type 1 diabetes. N Engl J Med 2010;363:311-320.

17. Chiarelli F, Marcovecchio LM: Type 1 diabetes: clinical and experimental; in Carel JC, Hochberg Z (eds): Yearbook of Pediatric Endocrinology 2011. Basel, Karger, 2011, pp 121-140.

18. Nguyen B, McGregor KA, O’Connor J, Shrewsbury VA, Lee A, Steinbeck KS, et al: Recruitment challenges and recommendations for adolescent obesity trials. J Paediatr Child Health 2012;48:38-43.

19. Norman RJ, Dewailly D, Legro RS, Hickey TE: Polycystic ovary syndrome. Lancet 2007;370:685-697.

20. Vlasselaers D, Milants I, Desmet L, Wouters PJ, Vanhorebeek I, van den Heuvel I, et al: Intensive insulin therapy for patients in paediatric intensive care: a prospective, randomised controlled study. Lancet 2009;373:547-556.

21. Jeschke MG, Kulp GA, Kraft R, Finnerty CC, Mlcak R, Lee JO, et al: Intensive insulin therapy in severely burned pediatric patients: a prospective randomized trial. Am J Respir Crit Care Med 2010;182:351-359.

22. Diamanti-Kandarakis E, Christakou CD, Kandaraki E, Economou FN: Metformin - an old medication of new fashion: evolving new molecular mechanisms and clinical implications in polycystic ovary syndrome. Eur J Endocrinol 2010;162:193-212.

23. Wang DS, Jonker JW, Kato Y, Kusuhara H, Schinkel AH, Sugiyama Y: Involvement of organic cation transporter 1 in hepatic and intestinal distribution of metformin. J Pharmacol Exp Ther 2002;302:510-515.

24. Shaw RJ, Lamia KA, Vasquez D, Koo SH, Bardeesy N, DePinho RA, et al: The kinase LKB1 mediates glucose homeostasis in liver and therapeutic effects of metformin. Science 2005;310:1642-1646.

25. Barber T, Bennett A, Groves C, Sovio U, Ruokonen A, Martikainen H, et al: Association of variants in the fat mass and obesity associated (FTO) gene with polycystic ovary syndrome. Diabetologia 2008;51:1153-1158. 
26. Xita N, Tsatsoulis A, Chatzikyriakidou A, Georgiou I: Association of the (TAAAA)n repeat polymorphism in the sex hormone-binding globulin (SHBG) gene with polycystic ovary syndrome and relation to SHBG serum levels. J Clin Endocrinol Metab 2003;88:5976-5980.

27. Ibáñez L, Ong KK, Mongan N, Jääskeläinen J, Marcos MV, Hughes IA, et al: Androgen receptor gene CAG repeat polymorphism in the development of ovarian hyperandrogenism. J Clin Endocrinol Metab 2003;88:3333-3338.

28. Juul A, Aksglaede L, Bay K, Grigor K Skakkebaek N (eds): Proceedings of the International Workshop on Klinefelter Syndrome 2010. Acta Paediatr 2011;100:791-792.

29. Kipling R: How the camel got his hump; in The Just So Stories. London, Macmillan, 1902.

30. Butler G: Evidence-based medicine in pediatric endocrinology; in Carel JC, Hochberg Z (eds): Yearbook of Pediatric Endocrinology 2010. Basel, Karger, 2010, pp 189-205. 